\title{
A modified genetic algorithm with a new crossover mating scheme
}

\author{
Allemar Jhone P. Delima ${ }^{1}$, Ariel M. Sison ${ }^{2}$, Ruji P. Medina ${ }^{3}$ \\ ${ }^{1,3}$ Graduate Programs, Technological Institute of the Philippines, Philippines \\ ${ }^{2}$ Emilio Aguinaldo College, Philippines
}

\begin{tabular}{l}
\hline Article Info \\
\hline Article historys: \\
Received Feb 20, 2019 \\
Revised Apr 11, 2019 \\
Accepted Apr 24, 2019 \\
\hline
\end{tabular}

Keywords:

Average crossover

Genetic algorithm

IBAX operator

Modified average crossover

Modified genetic algorithm

Novel crossover

\begin{abstract}
This study introduced the Inversed Bi-segmented Average Crossover (IBAX), a novel crossover operator that enhanced the offspring generation of the genetic algorithm (GA) for variable minimization and numerical optimization problems. An attempt to come up with a new mating scheme in generating new offspring under the crossover function through the novel IBAX operator has paved the way to a more efficient and optimized solution for variable minimization particularly on premature convergence problem using GA. A total of 597 records of student-respondents in the evaluation of the faculty instructional performance, represented by 30 variables, from the four State Universities and Colleges (SUC) in Caraga Region, Philippines was used as the dataset. The simulation results showed that the proposed modification on the Average Crossover (AX) of the genetic algorithm outperformed the genetic algorithm with the original AX operator. The GA with IBAX operator combined with rank-based selection function had removed 20 or $66.66 \%$ of the variables while 13 or $43.33 \%$ of the variables were removed when GA with AX operator and roulette wheel selection function was used.
\end{abstract}

Copyright (C) 2019 Institute of Advanced Engineering and Science. All rights reserved.

\section{Corresponding Author:}

Allemar Jhone P. Delima, Graduate Programs, Technological Institute of the Philippines, 938 Aurora Blvd., Cubao, Quezon City, Philippines.

Email: allemarjpd@ssct.edu.ph

\section{INTRODUCTION}

Data preprocessing [1-3] which is an imperative stride and considered to be one of the prime methods that is useful in data mining (DM), have led to the enhancement on the quality of data that positively contributes improvement to the precision and accuracy level as well as the mining efficiency of a prediction model $[4,5]$.

Data reduction, as an important data preprocessing technique in DM, is achieved through the selection and removal of unnecessary attributes and or variables in the dataset [6]. It is well known that in some cases, reducing original training set or variables by selecting the most representative information is advisable, yet obtaining nearly the same result or data-driven output [7-9]. Minimizing the size of the dataset aids in increasing the ability of generalization properties of the model. It also helped in lessening the space and computational time as well as minimizing the size of formulas used by the algorithm on the execution process [10]. Maximized accuracy through the reduced number of attributes $[11,6]$ and better understandability and interpretability of results are among the many benefits perceived in data reduction [12].

One of the competent data reduction, feature selection and global optimization algorithm that is widely used in related studies is the Genetic Algorithm [13-15]. Genetic Algorithms (GA), which was introduced by J.H. Holland in the 1970s, represents wide-ranging search method based on evolution and population genetics where its major executory mechanism relies on the crossover operator [16]. The unique integration of selection, crossover, and mutation operators serves as the driving force behind the successful 
implementation of GA. According to [17], the most widely-known problem in GAs is premature convergence. It occurs when genetic operators converge in an early stage after a few generations have been made and get stagnated there (local optima). Premature convergence occurs when the genetic operators cannot produce offspring that are a better representative of their parents whilst it is associated due to the loss of diversity in the population. According to [18], one of the technique to prevent premature convergence is to design an efficient crossover operator; thus, this study.

The activity that lies behind crossover is the creation of offspring that is achieved by combining information of the two parent chromosomes $[19,20]$. For real encoding problems using the arithmetic function, the average crossover (AX) [21] is modified in this study. The simplicity of the average crossover has opened an avenue for improvements for better genetic algorithm performance. The modification will solve the weakness of the GA since a new method of pairing genes from the chromosomes will be observed, and those other researchers may use it for their experimental parameters setting.

There is an appeal in the literature that encourages the enhancement of crossover operators for more effective optimization schemes of evolutionary algorithms. The influence of crossover operators is vital to the whole genetic algorithm process in the quest for optimal search space [22, 23].

Therefore, this study proposed a novel crossover operator as an enhancement to the average crossover of the genetic algorithm. The novel crossover is called Inversed Bi-segmented Average Crossover (IBAX) that alters the offspring generation of parents that are instrumental for the next generation. The rest of the paper is arranged as follows. Section 2 discusses the literature review of Genetic Algorithm. Section 3 includes the design and methodology used in the study. Section 4 discusses the results and discussions while Section 5 highlights the conclusion and recommendation.

\section{LITERATURE REVIEW}

\subsection{Genetic Algorithm}

Genetic algorithms as defined by [24], is one of the many evolutionary algorithms based on the rules of biological evolution for global optimization solution.

GA is known as one of the most competent and widely held techniques that are used to search the best or ideal solution for problems with a huge search space especially in combinatorial problems where the search space is of factorial order. GA produce and controls some individuals through the integration of various suitable generic operators to look for optimal solutions. The bottleneck for an optimal genetic algorithm implementation relies on its three fundamental operations after creating the initial population viz., selection, crossover, and mutation functions. Figure 1 shows the flowchart of the genetic algorithm.

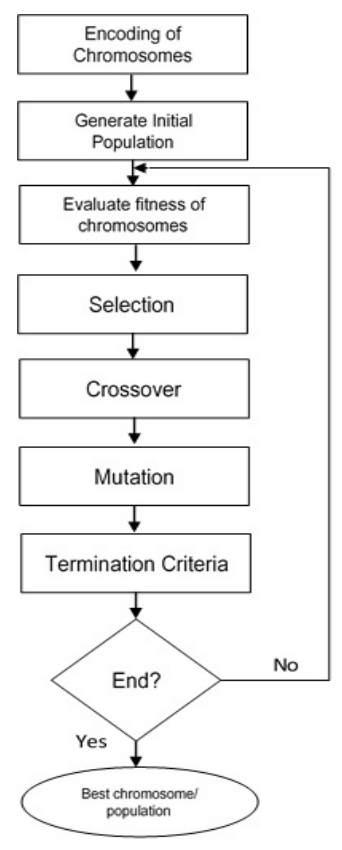

Figure 1. Genetic algorithm flowchart 


\subsubsection{Initialization / Evaluation of Fitness Function}

Fitness function serves as the backbone of the evaluation process of fitted values; hence, a vital step in GA execution. This serves as a performance determinant for relevant judgment [25].

\subsubsection{Selection}

This stage of the genetic algorithm is where the members in the population are selected to enter into the mating pool for the next function which is the crossover stage. The selection of an optimal operator for this stage is vital to ensure that members of the population who have higher fitness values can have a bigger chance of being selected for mating. Although, members with lower fitness function do still have a slim chance of being selected for reproduction. It is important to select the best members of the population to ensure that the search process is global and does not simply meet the nearest local optimum [26]. Selection is one of the important aspects of the GA process, and there are several ways for the selection as to wit: Binary Tournament Selection, Stochastic Universal Sampling (SUS), Roulette Wheel Selection (RWS), Elitism Selection, and Rank-based Selection. For the detailed explanation of the abovementioned selection schemes, the study of [27] is recommended. Below are the following selection functions used in this study:

\section{- Roulette Wheel Selection (RWS) Function}

According to [28], Roulette selection is one of the simplest traditional GA selection technique. To execute, all the chromosomes in the population are placed on the roulette wheel according to their fitness value. A segment is assigned as representation to each individual commensurate to their fitness value; hence, the bigger the fitness value is, the larger the segment. Then, the virtual roulette wheel is spun. The individual corresponding to the segment on which roulette wheel stops are then selected. The process is repeated until the desired number of individuals is selected. Individuals with higher fitness have more probability of selection.

\section{- Rank-based Selection Function}

The rank-based selection function according to [29] can be assigned depending on the distribution of chromosomes according to their fitness values. This can be executed through positioning the chromosomes in decreasing order according to their fitness values. Next is to allocate a rank value on every chromosome

That corresponds to its arrangement in the set and then calculate the new fitness value for every chromosome using (1):

$$
F=\max -(\max -\min ) * \frac{\operatorname{rank}-1}{\text { Npop }-1}
$$

where $1<\max <=2 \& \min =2-\max$

\subsubsection{Crossover}

The Crossover is identified to be the most important operator in genetic algorithms. It is responsible for generating new offspring that will be used for the next generation by combining features of two parent chromosomes [21].

A recent study was conducted by [22] that enhanced the Average Crossover (AX) operator of the genetic algorithm. The proposed operator is called Cross Average Crossover (CAX). The use of the modified genetic algorithm with CAX operator and rank-based selection function yielded to more decreased variables than the traditional genetic algorithm, but a degradation phenomenon [30] was depicted. The CAX operator with rank-based selection function eliminated those individuals with higher fitness values due to the structure of its mating scheme.

According to [19], there are two categories of crossover development. They are called parent-centric and mean centric operators. The parent-centric approach generates offspring within the vicinity of each of the parent chromosomes while the mean centric generates offspring solutions by identifying the central tendencies of the parents involved. The Average Crossover which is a well-known crossover operator for real encoding problems found in the study of [21], that is modified this study, is outlined below along with the CAX operator:

\section{- Average Crossover (AX)}

Part or all of the genes are averages of the same alleles in both the parents. Select two parallel parents and compute its average to create offspring.

\section{- Cross Average Crossover (CAX)}

A modified version of Average Crossover (AX) where the first gene in the first chromosome and the last gene of the second chromosome are averaged and get its value. The resulting average values are considered as offspring. Repeat the steps until genes from chromosomes have crossed in creating offspring. 


\subsubsection{Mutation}

Studies have been carried out on the varieties of mutation techniques to improve GAs performance over the years. The purpose of mutation operation is to change the genes of the offspring and to increase the diversity of the population. This process enables GAs to jump out of local or suboptimal solutions to avoid premature convergence [19].

\section{METHODOLOGY}

In this study, the average crossover which is one of the crossover operators in the genetic algorithm is modified. The use of the roulette wheel and rank-based selection function were observed. The variables who obtained the lowest fitness function in each generation for ten generations were removed. Instead of pairing the parallel genes from chromosomes $\mathrm{x}$ and $\mathrm{y}$ and compute its average to produce offspring $\mathrm{z}$ as shown in Figure 2, it is suggested to segment the chromosomes ( $\mathrm{x}$ and $\mathrm{y}$ ) into two and inversely compute the average of genes within each segment created as depicted in Figure 3. The modified crossover will be called Inversed Bisegmented Average Crossover (IBAX).

\subsection{Existing Traditional Average Crossover}

The average crossover is simple and can be implemented through the following steps:

Step 1: Take two parents from the selection pool.

Step 2: Create offspring $\mathrm{Z}$ from two parallel parents $\mathrm{X}$ and $\mathrm{Y}$

Step 3: Use the formula

$$
z=[x+y] / 2
$$

Step 4: For $\mathrm{i}=1$ to $n$ do formula (2)

Step 5: End do

\begin{tabular}{|c|c|c|}
\hline$x$ & & $Y$ \\
\hline 545 & 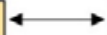 & 535 \\
\hline 541 & $4 \longrightarrow$ & 528 \\
\hline 537 & $\longleftrightarrow$ & 545 \\
\hline 535 & 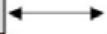 & 541 \\
\hline 528 & $\hookrightarrow$ & 537 \\
\hline 528 & 4 & 523 \\
\hline 525 & $\longleftrightarrow$ & 520 \\
\hline 525 & $\longleftrightarrow$ & 528 \\
\hline 523 & $\hookrightarrow$ & 525 \\
\hline 520 & $\longleftrightarrow$ & 525 \\
\hline 518 & $\leftrightarrow$ & 518 \\
\hline
\end{tabular}

Figure 2. Average crossover with roulette wheel selection function

\subsection{Modified Average Crossover}

For the IBAX operator to be realized, the following steps must be executed:

Step 1: Take the parents from the selection pool.

Step 2: Count the number of genes found in the chromosomes. Identify if the dataset is in odd or even numbers.

Step 3: Segment the chromosomes ( $\mathrm{x}$ and $\mathrm{y}$ ) by dividing the total number of genes in the chromosomes into two and make sure that both first and second segments must contain an equal number of genes in an even count.

Step 4: On the first segment, create offspring $\mathrm{Z}$ for each gene by inversely pairing the first gene from chromosome $\mathrm{X}$ to the last gene on chromosome $\mathrm{Y}$. Repeat until the last gene of the chromosome $\mathrm{X}$ and the first gene of the chromosome $\mathrm{Y}$ have inversely mated and have produced an offspring using formula (2).

Step 5: Execute the same process on the second segment until genes from all segments have produced offspring. In the case of odd datasets, the last genes of the chromosomes will not be combined in the second segment and will automatically be mated with each other to produce offspring.

Indo. J. Elec. Eng. \& Inf, Vol.7, No. 2, June 2019: 165 - 181 


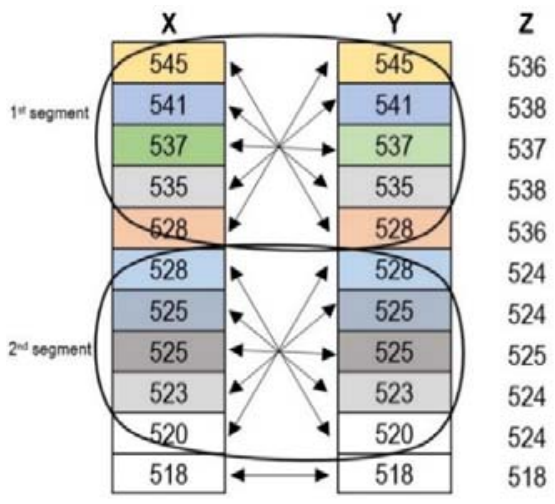

Figure 3. Inversed Bi-segmented Average Crossover with the rank-based selection function

\subsection{Datasets}

In this study, a total of 597 records of student-respondents in the evaluation of the faculty instructional performance from the four State Universities and Colleges (SUC) in Caraga Region, Philippines were used as the datasets. There were thirty (30) variables that represent the faculty instructional performance having divided into six (6) parts viz., methodology, classroom management, student discipline, assessment of learning, student-teacher relationship, and peer relationship. Each category has five items as shown in Table 1.

Table 1. Variables used in the study

\begin{tabular}{|c|c|c|c|}
\hline Category & Reasons & Variable & Possible Value \\
\hline \multirow{7}{*}{ Methodology } & $\begin{array}{l}\text { Utilizes varied designs/ techniques/ activities suited to the } \\
\text { different types of learners. }\end{array}$ & M1 & $\{1,2,3\}$ \\
\hline & $\begin{array}{l}\text { Explains learning goals and instructional procedures to the } \\
\text { students. }\end{array}$ & M2 & $\{1,2,3\}$ \\
\hline & $\begin{array}{l}\text { Uses real-life examples in the class to sustain student's } \\
\text { interest in learning. }\end{array}$ & M3 & $\{1,2,3\}$ \\
\hline & $\begin{array}{l}\text { Creates a situation that encourages students to use critical } \\
\text { thinking. }\end{array}$ & M4 & $\{1,2,3\}$ \\
\hline & Delivers accurate/relevant/updated content knowledge. & M5 & $\{1,2,3\}$ \\
\hline & Establishes routines to maximize instructional time. & $\mathrm{C} 1$ & $\{1,2,3\}$ \\
\hline & Organizes and assign the daily cleaners. & $\mathrm{C} 2$ & $\{1,2,3\}$ \\
\hline \multirow{2}{*}{$\begin{array}{l}\text { Classroom } \\
\text { Management }\end{array}$} & Employs an effective system of classroom set-up. & $\mathrm{C} 3$ & $\{1,2,3\}$ \\
\hline & $\begin{array}{l}\text { Employs strategies to maximize the use of resources in } \\
\text { learning activities. }\end{array}$ & $\mathrm{C} 4$ & $\{1,2,3\}$ \\
\hline \multirow{6}{*}{$\begin{array}{l}\text { Student } \\
\text { Discipline }\end{array}$} & Implements rules/policies inside the classroom. & C5 & $\{1,2,3\}$ \\
\hline & Handles behavior problem concerning the student's rights. & SD1 & $\{1,2,3\}$ \\
\hline & $\begin{array}{l}\text { Imposes disciplinary sanction(s) to the misbehaving } \\
\text { student(s). }\end{array}$ & SD2 & $\{1,2,3\}$ \\
\hline & Encourages students to submit requirements on time. & SD3 & $\{1,2,3\}$ \\
\hline & Motivates students to respect each other. & SD4 & $\{1,2,3\}$ \\
\hline & Allows students to exercise their creativity. & SD5 & $\{1,2,3\}$ \\
\hline \multirow{5}{*}{$\begin{array}{l}\text { Assessment } \\
\text { of Learning }\end{array}$} & Constructs valid and reliable formative and summative tests. & A1 & $\{1,2,3\}$ \\
\hline & $\begin{array}{l}\text { Uses appropriate non-traditional assessment techniques and } \\
\text { tools (i.e. portfolio, journals, rubric, etc) }\end{array}$ & A2 & $\{1,2,3\}$ \\
\hline & $\begin{array}{l}\text { Interprets and use test results to improve teaching and } \\
\text { learning. }\end{array}$ & A3 & $\{1,2,3\}$ \\
\hline & Uses tools for assessing authentic learning. & A4 & $\{1,2,3\}$ \\
\hline & Provides timely and accurate feedback to students. & A5 & $\{1,2,3\}$ \\
\hline \multirow{5}{*}{$\begin{array}{l}\text { Student- } \\
\text { teacher } \\
\text { relationship }\end{array}$} & $\begin{array}{l}\text { Encourages students to participate in class/school activities } \\
\text { actively. }\end{array}$ & ST1 & $\{1,2,3\}$ \\
\hline & Allows students to communicate directly to $\mathrm{him} / \mathrm{her}$. & ST2 & $\{1,2,3\}$ \\
\hline & Provides equal opportunities for all students. & ST3 & $\{1,2,3\}$ \\
\hline & Promotes teamwork among students. & ST4 & $\{1,2,3\}$ \\
\hline & Makes him/herself available to students. & ST5 & $\{1,2,3\}$ \\
\hline \multirow{5}{*}{$\begin{array}{l}\text { Peer } \\
\text { relationship }\end{array}$} & $\begin{array}{l}\text { Demonstrates appropriate behavior in dealing with } \\
\text { students/peers/superiors. }\end{array}$ & P1 & $\{1,2,3\}$ \\
\hline & Manifests flexibility when deemed necessary. & $\mathrm{P} 2$ & $\{1,2,3\}$ \\
\hline & Exhibits collegiality with colleagues. & P3 & $\{1,2,3\}$ \\
\hline & Observes professionalism at all times. & $\mathrm{P} 4$ & $\{1,2,3\}$ \\
\hline & Empathizes other needs and concern. & P5 & $\{1,2,3\}$ \\
\hline
\end{tabular}




\section{RESULTS AND DISCUSSION}

\subsection{Simulation result for GA with AX operator and RWS Function}

The simulation on the genetic algorithm was done for ten generations utilizing the existing traditional average crossover and roulette wheel selection function. The 597 records of random student-respondents in the evaluation of the faculty instructional performance (IP) from the four State Universities and Colleges (SUC) in Caraga Region, Philippines were instrumental in this study.

First Generation: Variable $\mathrm{C} 2$ is removed from the chromosome since it obtained the lowest fitness value of 171396 as evident in Table 2.

Second Generation: Variables M5 and A2 were removed from the chromosome since both obtained the lowest fitness value of 263169 as evident in Table 3.

Table 2. G1 using an average crossover with RWS function

\begin{tabular}{|c|c|c|c|c|c|c|c|c|}
\hline \multirow{2}{*}{ IP } & \multirow{2}{*}{$X$} & \multirow{2}{*}{ Fitness } & \multirow{2}{*}{ Rank } & \multicolumn{2}{|c|}{ Pool } & \multirow{2}{*}{ Off-spring } & \multirow{2}{*}{ Fitness } & \multirow{2}{*}{ Decision } \\
\hline & & & & Y & IP & & & \\
\hline M1 & 546 & 298116 & 22 & 552 & M5 & 549 & 301401 & \\
\hline M2 & 565 & 319225 & 30 & 565 & M2 & 565 & 319225 & \\
\hline M3 & 558 & 311364 & 27 & 548 & SD1 & 553 & 305809 & \\
\hline M4 & 559 & 312481 & 28 & 546 & SD5 & 552.5 & 305256.3 & \\
\hline M5 & 552 & 304704 & 24 & 474 & C3 & 513 & 263169 & \\
\hline $\mathrm{C} 1$ & 490 & 240100 & 3 & 546 & A3 & 518 & 268324 & \\
\hline $\mathrm{C} 2$ & 354 & 125316 & 1 & 474 & C3 & 414 & 171396 & Removt \\
\hline $\mathrm{C} 3$ & 474 & 224676 & 2 & 556 & ST1 & 515 & 265225 & \\
\hline $\mathrm{C} 4$ & 542 & 293764 & 18 & 490 & $\mathrm{C} 1$ & 516 & 266256 & \\
\hline $\mathrm{C} 5$ & 528 & 278784 & 12 & 531 & ST3 & 529.5 & 280370.3 & \\
\hline SD1 & 548 & 300304 & 23 & 500 & A2 & 524 & 274576 & \\
\hline SD2 & 512 & 262144 & 5 & 542 & $\mathrm{C} 4$ & 527 & 277729 & \\
\hline SD3 & 565 & 319225 & 29 & 546 & A3 & 555.5 & 308580.3 & \\
\hline SD4 & 556 & 309136 & 26 & 558 & M3 & 557 & 310249 & \\
\hline SD5 & 546 & 298116 & 21 & 528 & $\mathrm{C} 5$ & 537 & 288369 & \\
\hline A1 & 513 & 263169 & 6 & 534 & ST2 & 523.5 & 274052.3 & \\
\hline $\mathrm{A} 2$ & 500 & 250000 & 4 & 526 & P3 & 513 & 263169 & \\
\hline A3 & 546 & 298116 & 20 & 513 & A1 & 529.5 & 280370.3 & \\
\hline A4 & 518 & 268324 & 8 & 565 & M2 & 541.5 & 293222.3 & \\
\hline A5 & 516 & 266256 & 7 & 516 & A5 & 516 & 266256 & \\
\hline ST1 & 556 & 309136 & 25 & 556 & ST1 & 556 & 309136 & \\
\hline ST2 & 534 & 285156 & 16 & 559 & M4 & 546.5 & 298662.3 & \\
\hline ST3 & 531 & 281961 & 14 & 546 & M1 & 538.5 & 289982.3 & \\
\hline ST4 & 541 & 292681 & 17 & 556 & SD4 & 548.5 & 300852.3 & \\
\hline ST5 & 527 & 277729 & 11 & 552 & M5 & 539.5 & 291060.3 & \\
\hline $\mathrm{P} 1$ & 531 & 281961 & 13 & 565 & SD3 & 548 & 300304 & \\
\hline P2 & 533 & 284089 & 15 & 541 & ST4 & 537 & 288369 & \\
\hline P3 & 526 & 276676 & 10 & 518 & A4 & 522 & 272484 & \\
\hline P4 & 526 & 276676 & 9 & 565 & SD3 & 545.5 & 297570.3 & \\
\hline P5 & 544 & 295936 & 19 & 565 & SD3 & 554.5 & 307470.3 & \\
\hline
\end{tabular}

Table 3. G2 using an average crossover with RWS function

\begin{tabular}{ccccccccc}
\hline \multirow{2}{*}{ IP } & \multirow{2}{*}{ X } & \multirow{2}{*}{ Fitness } & Rank & Y & IP & Off-spring & Fitness & \multirow{2}{*}{ Decision } \\
\hline M1 & 546 & 298116 & 22 & 552 & M5 & 549 & 301401 & \\
M2 & 565 & 319225 & 30 & 565 & M2 & 565 & 319225 & \\
M3 & 558 & 311364 & 27 & 548 & SD1 & 553 & 305809 & \\
M4 & 559 & 312481 & 28 & 546 & SD5 & 552.5 & 305256.3 & \\
M5 & 552 & 304704 & 24 & 474 & C3 & 513 & 263169 & Remove \\
C1 & 490 & 240100 & 3 & 546 & A3 & 518 & 268324 & \\
C3 & 474 & 224676 & 2 & 556 & ST1 & 515 & 265225 & \\
C4 & 542 & 293764 & 18 & 490 & C1 & 516 & 266256 & \\
C5 & 528 & 278784 & 12 & 531 & ST3 & 529.5 & 280370.3 & \\
SD1 & 548 & 300304 & 23 & 500 & A2 & 524 & 274576 & \\
SD2 & 512 & 262144 & 5 & 542 & C4 & 527 & 277729 & \\
SD3 & 565 & 319225 & 29 & 546 & A3 & 555.5 & 308580.3 & \\
SD4 & 556 & 309136 & 26 & 558 & M3 & 557 & 310249 & \\
SD5 & 546 & 298116 & 21 & 528 & C5 & 537 & 288369 & \\
A1 & 513 & 263169 & 6 & 534 & ST2 & 523.5 & 274052.3 & \\
A2 & 500 & 250000 & 4 & 526 & P3 & 513 & 263169 & Remove \\
A3 & 546 & 298116 & 20 & 513 & A1 & 529.5 & 280370.3 & \\
\hline
\end{tabular}


Indo. J. Elec. Eng. \& Inf

ISSN: 2089-3272

171

\begin{tabular}{ccccccccc}
\hline \multirow{2}{*}{ IP } & \multirow{2}{*}{ X } & Fitness & \multirow{2}{*}{ Rank } & Y & IP & Off-spring & Fitness & \multirow{2}{*}{ Decision } \\
\hline A4 & 518 & 268324 & 8 & 565 & M2 & 541.5 & 293222.3 & \\
A5 & 516 & 266256 & 7 & 516 & A5 & 516 & 266256 & \\
ST1 & 556 & 309136 & 25 & 556 & ST1 & 556 & 309136 & \\
ST2 & 534 & 285156 & 16 & 559 & M4 & 546.5 & 298662.3 & \\
ST3 & 531 & 281961 & 14 & 546 & M1 & 538.5 & 289982.3 & \\
ST4 & 541 & 292681 & 17 & 556 & SD4 & 548.5 & 300852.3 & \\
ST5 & 527 & 277729 & 11 & 552 & M5 & 539.5 & 291060.3 & \\
P1 & 531 & 281961 & 13 & 565 & SD3 & 548 & 300304 & \\
P2 & 533 & 284089 & 15 & 541 & ST4 & 537 & 288369 & \\
P3 & 526 & 276676 & 10 & 518 & A4 & 522 & 272484 & \\
P4 & 526 & 276676 & 9 & 565 & SD3 & 545.5 & 297570.3 & \\
P5 & 544 & 295936 & 19 & 565 & SD3 & 554.5 & 307470.3 & \\
\hline
\end{tabular}

Third Generation: Variable C3 is removed from the chromosome since it obtained the lowest fitness value of 265225 as evident in Table 4.

Fourth Generation: Variables C4 and A5 were removed from the chromosome since it obtained the lowest fitness value of 266256 as evident in Table 5 .

Table 4. G3 using an average crossover with RWS function

\begin{tabular}{ccccccccc}
\hline \multirow{2}{*}{ IP } & X & Fitness & Rank & Y & IP & Off-spring & Fitness & \multirow{2}{*}{ Decision } \\
\hline M1 & 546 & 298116 & 22 & 552 & M5 & 549 & 301401 & \\
M2 & 565 & 319225 & 30 & 565 & M2 & 565 & 319225 & \\
M3 & 558 & 311364 & 27 & 548 & SD1 & 553 & 305809 & \\
M4 & 559 & 312481 & 28 & 546 & SD5 & 552.5 & 305256.3 & \\
C1 & 490 & 240100 & 3 & 546 & A3 & 518 & 268324 & \\
C3 & 474 & 224676 & 2 & 556 & ST1 & 515 & 265225 & Remove \\
C4 & 542 & 293764 & 18 & 490 & C1 & 516 & 266256 & \\
C5 & 528 & 278784 & 12 & 531 & ST3 & 529.5 & 280370.3 & \\
SD1 & 548 & 300304 & 23 & 500 & A2 & 524 & 274576 & \\
SD2 & 512 & 262144 & 5 & 542 & C4 & 527 & 277729 & \\
SD3 & 565 & 319225 & 29 & 546 & A3 & 555.5 & 308580.3 & \\
SD4 & 556 & 309136 & 26 & 558 & M3 & 557 & 310249 & \\
SD5 & 546 & 298116 & 21 & 528 & C5 & 537 & 288369 & \\
A1 & 513 & 263169 & 6 & 534 & ST2 & 523.5 & 274052.3 & \\
A3 & 546 & 298116 & 20 & 513 & A1 & 529.5 & 280370.3 & \\
A4 & 518 & 268324 & 8 & 565 & M2 & 541.5 & 293222.3 & \\
A5 & 516 & 266256 & 7 & 516 & A5 & 516 & 266256 & \\
ST1 & 556 & 309136 & 25 & 556 & ST1 & 556 & 309136 & \\
ST2 & 534 & 285156 & 16 & 559 & M4 & 546.5 & 298662.3 & \\
ST3 & 531 & 281961 & 14 & 546 & M1 & 538.5 & 289982.3 & \\
ST4 & 541 & 292681 & 17 & 556 & SD4 & 548.5 & 300852.3 & \\
ST5 & 527 & 277729 & 11 & 552 & M5 & 539.5 & 291060.3 & \\
P1 & 531 & 281961 & 13 & 565 & SD3 & 548 & 300304 & \\
P2 & 533 & 284089 & 15 & 541 & ST4 & 537 & 288369 & \\
P3 & 526 & 276676 & 10 & 518 & A4 & 522 & 272484 & \\
P4 & 526 & 276676 & 9 & 565 & SD3 & 545.5 & 297570.3 & \\
P5 & 544 & 295936 & 19 & 565 & SD3 & 554.5 & 307470.3 & \\
\hline
\end{tabular}

Table 5. G4 using an average crossover with RWS function

\begin{tabular}{ccccccccc}
\hline \multirow{2}{*}{ IP } & \multirow{2}{*}{ X } & \multirow{2}{*}{ Fitness } & \multirow{2}{*}{ Rank } & Y & IP & Off-spring & \multirow{2}{*}{ Fitness } & \multirow{2}{*}{ Decision } \\
\hline 1 & 546 & 98116 & 2 & 52 & 5 & 549 & 301401 & \\
M2 & 565 & 319225 & 30 & 565 & M2 & 565 & 319225 & \\
M3 & 558 & 311364 & 27 & 548 & SD1 & 553 & 305809 & \\
M4 & 559 & 312481 & 28 & 546 & SD5 & 552.5 & 305256.3 & \\
C1 & 490 & 240100 & 3 & 546 & A3 & 518 & 268324 & \\
C4 & 542 & 293764 & 18 & 490 & C1 & 516 & 266256 & Remove \\
C5 & 528 & 278784 & 12 & 531 & ST3 & 529.5 & 280370.3 & \\
SD1 & 548 & 300304 & 23 & 500 & A2 & 524 & 274576 & \\
SD2 & 512 & 262144 & 5 & 542 & C4 & 527 & 277729 & \\
SD3 & 565 & 319225 & 29 & 546 & A3 & 555.5 & 308580.3 & \\
SD4 & 556 & 309136 & 26 & 558 & M3 & 557 & 310249 & \\
SD5 & 546 & 298116 & 21 & 528 & C5 & 537 & 288369 & \\
A1 & 513 & 263169 & 6 & 534 & ST2 & 523.5 & 274052.3 & \\
A3 & 546 & 298116 & 20 & 513 & A1 & 529.5 & 280370.3 & \\
\hline
\end{tabular}

A modified genetic algorithm with a new crossover mating scheme (Allemar Jhone P. Delima) 


\begin{tabular}{ccccccccc}
\hline \multirow{2}{*}{ IP } & \multirow{2}{*}{ X } & \multirow{2}{*}{ Fitness } & \multirow{2}{*}{ Rank } & Y & IP & Off-spring & \multirow{2}{*}{ Fitness } & \multirow{2}{*}{ Decision } \\
\hline A4 & 518 & 268324 & 8 & 565 & M2 & 541.5 & 293222.3 & \\
A5 & 516 & 266256 & 7 & 516 & A5 & 516 & 266256 & Remove \\
ST1 & 556 & 309136 & 25 & 556 & ST1 & 556 & 309136 & \\
ST2 & 534 & 285156 & 16 & 559 & M4 & 546.5 & 298662.3 & \\
ST3 & 531 & 281961 & 14 & 546 & M1 & 538.5 & 289982.3 & \\
ST4 & 541 & 292681 & 17 & 556 & SD4 & 548.5 & 300852.3 & \\
ST5 & 527 & 277729 & 11 & 552 & M5 & 539.5 & 291060.3 & \\
P1 & 531 & 281961 & 13 & 565 & SD3 & 548 & 300304 & \\
P2 & 533 & 284089 & 15 & 541 & ST4 & 537 & 288369 & \\
P3 & 526 & 276676 & 10 & 518 & A4 & 522 & 272484 & \\
P4 & 526 & 276676 & 9 & 565 & SD3 & 545.5 & 297570.3 & \\
P5 & 544 & 295936 & 19 & 565 & SD3 & 554.5 & 307470.3 & \\
\hline
\end{tabular}

Fifth Generation: Variable $\mathrm{C} 1$ is removed from the chromosome since it obtained the lowest fitness value of 268324 as evident in Table 6.

Sixth Generation: Variable P3 is removed from the chromosome since it obtained the lowest fitness value of 272484 as evident in Table 7.

Table 6. G5 using an average crossover with RWS function

\begin{tabular}{ccccccccc}
\hline \multirow{2}{*}{ IP } & X & \multirow{2}{*}{ Fitness } & \multirow{2}{*}{ Rank } & Y & IP & Off-spring & Fitness & \multirow{2}{*}{ Decision } \\
\hline M1 & 546 & 298116 & 22 & 552 & M5 & 549 & 301401 & \\
M2 & 565 & 319225 & 30 & 565 & M2 & 565 & 319225 & \\
M3 & 558 & 311364 & 27 & 548 & SD1 & 553 & 305809 & \\
M4 & 559 & 312481 & 28 & 546 & SD5 & 552.5 & 305256.3 & \\
C1 & 490 & 240100 & 3 & 546 & A3 & 518 & 268324 & Remove \\
C5 & 528 & 278784 & 12 & 531 & ST3 & 529.5 & 280370.3 & \\
SD1 & 548 & 300304 & 23 & 500 & A2 & 524 & 274576 & \\
SD2 & 512 & 262144 & 5 & 542 & C4 & 527 & 277729 & \\
SD3 & 565 & 319225 & 29 & 546 & A3 & 555.5 & 308580.3 & \\
SD4 & 556 & 309136 & 26 & 558 & M3 & 557 & 310249 & \\
SD5 & 546 & 298116 & 21 & 528 & C5 & 537 & 288369 & \\
A1 & 513 & 263169 & 6 & 534 & ST2 & 523.5 & 274052.3 & \\
A3 & 546 & 298116 & 20 & 513 & A1 & 529.5 & 280370.3 & \\
A4 & 518 & 268324 & 8 & 565 & M2 & 541.5 & 293222.3 & \\
ST1 & 556 & 309136 & 25 & 556 & ST1 & 556 & 309136 & \\
ST2 & 534 & 285156 & 16 & 559 & M4 & 546.5 & 298662.3 & \\
ST3 & 531 & 281961 & 14 & 546 & M1 & 538.5 & 289982.3 & \\
ST4 & 541 & 292681 & 17 & 556 & SD4 & 548.5 & 300852.3 & \\
ST5 & 527 & 277729 & 11 & 552 & M5 & 539.5 & 291060.3 & \\
P1 & 531 & 281961 & 13 & 565 & SD3 & 548 & 300304 & \\
P2 & 533 & 284089 & 15 & 541 & ST4 & 537 & 288369 & \\
P3 & 526 & 276676 & 10 & 518 & A4 & 522 & 272484 & \\
P4 & 526 & 276676 & 9 & 565 & SD3 & 545.5 & 297570.3 & \\
P5 & 544 & 295936 & 19 & 565 & SD3 & 554.5 & 307470.3 & \\
\hline
\end{tabular}

Table 7. G6 using an average crossover with RWS function

\begin{tabular}{ccccccccc}
\hline \multirow{2}{*}{ IP } & X & Fitness & Rank & Y & IP & Off-spring & Fitness & \multirow{2}{*}{ Decision } \\
\hline M1 & 546 & 298116 & 22 & 552 & M5 & 549 & 301401 & \\
M2 & 565 & 319225 & 30 & 565 & M2 & 565 & 319225 & \\
M3 & 558 & 311364 & 27 & 548 & SD1 & 553 & 305809 & \\
M4 & 559 & 312481 & 28 & 546 & SD5 & 552.5 & 305256.3 & \\
C5 & 528 & 278784 & 12 & 531 & ST3 & 529.5 & 280370.3 & \\
SD1 & 548 & 300304 & 23 & 500 & A2 & 524 & 274576 & \\
SD2 & 512 & 262144 & 5 & 542 & C4 & 527 & 277729 & \\
SD3 & 565 & 319225 & 29 & 546 & A3 & 555.5 & 308580.3 & \\
SD4 & 556 & 309136 & 26 & 558 & M3 & 557 & 310249 & \\
SD5 & 546 & 298116 & 21 & 528 & C5 & 537 & 288369 & \\
A1 & 513 & 263169 & 6 & 534 & ST2 & 523.5 & 274052.3 & \\
A3 & 546 & 298116 & 20 & 513 & A1 & 529.5 & 280370.3 & \\
A4 & 518 & 268324 & 8 & 565 & M2 & 541.5 & 293222.3 & \\
ST1 & 556 & 309136 & 25 & 556 & ST1 & 556 & 309136 & \\
ST2 & 534 & 285156 & 16 & 559 & M4 & 546.5 & 298662.3 & \\
ST3 & 531 & 281961 & 14 & 546 & M1 & 538.5 & 289982.3 & \\
ST4 & 541 & 292681 & 17 & 556 & SD4 & 548.5 & 300852.3 & \\
\hline
\end{tabular}


Indo. J. Elec. Eng. \& Inf

ISSN: $2089-3272$

\begin{tabular}{ccccccccr}
\hline \multirow{2}{*}{ IP } & \multirow{2}{*}{ X } & \multirow{2}{*}{ Fitness } & \multirow{2}{*}{ Rank } & Y & IP & Off-spring & \multirow{2}{*}{ Fitness } & Decision \\
\hline ST5 & 527 & 277729 & 11 & 552 & M5 & 539.5 & 291060.3 & \\
P1 & 531 & 281961 & 13 & 565 & SD3 & 548 & 300304 & \\
P2 & 533 & 284089 & 15 & 541 & ST4 & 537 & 288369 & \\
P3 & 526 & 276676 & 10 & 518 & A4 & 522 & 272484 & Remove \\
P4 & 526 & 276676 & 9 & 565 & SD3 & 545.5 & 297570.3 & \\
P5 & 544 & 295936 & 19 & 565 & SD3 & 554.5 & 307470.3 & \\
\hline
\end{tabular}

Seventh Generation: Variable A1 is removed from the chromosome since it obtained the lowest fitness value of 274052.3 as evident in Table 8 .

Table 8. G7 using an average crossover with RWS function

\begin{tabular}{ccccccccc}
\hline \multirow{2}{*}{ IP } & \multirow{2}{*}{ X } & \multirow{2}{*}{ Fitness } & Rank & Y & IP & Off-spring & \multirow{2}{*}{ Fitness } & \multirow{2}{*}{ Decision } \\
\hline M1 & 546 & 298116 & 22 & 552 & M5 & 549 & 301401 & \\
M2 & 565 & 319225 & 30 & 565 & M2 & 565 & 319225 & \\
M3 & 558 & 311364 & 27 & 548 & SD1 & 553 & 305809 & \\
M4 & 559 & 312481 & 28 & 546 & SD5 & 552.5 & 305256.3 & \\
C5 & 528 & 278784 & 12 & 531 & ST3 & 529.5 & 280370.3 & \\
SD1 & 548 & 300304 & 23 & 500 & A2 & 524 & 274576 & \\
SD2 & 512 & 262144 & 5 & 542 & C4 & 527 & 277729 & \\
SD3 & 565 & 319225 & 29 & 546 & A3 & 555.5 & 308580.3 & \\
SD4 & 556 & 309136 & 26 & 558 & M3 & 557 & 310249 & \\
SD5 & 546 & 298116 & 21 & 528 & C5 & 537 & 288369 & \\
A1 & 513 & 263169 & 6 & 534 & ST2 & 523.5 & 274052.3 & Remove \\
A3 & 546 & 298116 & 20 & 513 & A1 & 529.5 & 280370.3 & \\
A4 & 518 & 268324 & 8 & 565 & M2 & 541.5 & 293222.3 & \\
ST1 & 556 & 309136 & 25 & 556 & ST1 & 556 & 309136 & \\
ST2 & 534 & 285156 & 16 & 559 & M4 & 546.5 & 298662.3 & \\
ST3 & 531 & 281961 & 14 & 546 & M1 & 538.5 & 289982.3 & \\
ST4 & 541 & 292681 & 17 & 556 & SD4 & 548.5 & 300852.3 & \\
ST5 & 527 & 277729 & 11 & 552 & M5 & 539.5 & 291060.3 & \\
P1 & 531 & 281961 & 13 & 565 & SD3 & 548 & 300304 & \\
P2 & 533 & 284089 & 15 & 541 & ST4 & 537 & 288369 & \\
P4 & 526 & 276676 & 9 & 565 & SD3 & 545.5 & 297570.3 & \\
P5 & 544 & 295936 & 19 & 565 & SD3 & 554.5 & 307470.3 & \\
\hline
\end{tabular}

Eight Generation: Variable SD1 is removed from the chromosome since it obtained the lowest fitness value of 274576 as evident in Table 9.

Table 9. G8 using an average crossover with RWS function

\begin{tabular}{ccccccccc}
\hline \multirow{2}{*}{ IP } & \multirow{2}{*}{ X } & \multirow{2}{*}{ Fitness } & \multirow{2}{*}{ Rank } & Y & IP & Off-spring & Fitness & \multirow{2}{*}{ Decision } \\
\hline M1 & 546 & 298116 & 22 & 552 & M5 & 549 & 301401 & \\
M2 & 565 & 319225 & 30 & 565 & M2 & 565 & 319225 & \\
M3 & 558 & 311364 & 27 & 548 & SD1 & 553 & 305809 & \\
M4 & 559 & 312481 & 28 & 546 & SD5 & 552.5 & 305256.3 & \\
C5 & 528 & 278784 & 12 & 531 & ST3 & 529.5 & 280370.3 & \\
SD1 & 548 & 300304 & 23 & 500 & A2 & 524 & 274576 & Remove \\
SD2 & 512 & 262144 & 5 & 542 & C4 & 527 & 277729 & \\
SD3 & 565 & 319225 & 29 & 546 & A3 & 555.5 & 308580.3 & \\
SD4 & 556 & 309136 & 26 & 558 & M3 & 557 & 310249 & \\
SD5 & 546 & 298116 & 21 & 528 & C5 & 537 & 288369 & \\
A3 & 546 & 298116 & 20 & 513 & A1 & 529.5 & 280370.3 & \\
A4 & 518 & 268324 & 8 & 565 & M2 & 541.5 & 293222.3 & \\
ST1 & 556 & 309136 & 25 & 556 & ST1 & 556 & 309136 & \\
ST2 & 534 & 285156 & 16 & 559 & M4 & 546.5 & 298662.3 & \\
ST3 & 531 & 281961 & 14 & 546 & M1 & 538.5 & 289982.3 & \\
ST4 & 541 & 292681 & 17 & 556 & SD4 & 548.5 & 300852.3 & \\
ST5 & 527 & 277729 & 11 & 552 & M5 & 539.5 & 291060.3 & \\
P1 & 531 & 281961 & 13 & 565 & SD3 & 548 & 300304 & \\
P2 & 533 & 284089 & 15 & 541 & ST4 & 537 & 288369 & \\
P4 & 526 & 276676 & 9 & 565 & SD3 & 545.5 & 297570.3 & \\
P5 & 544 & 295936 & 19 & 565 & SD3 & 554.5 & 307470.3 & \\
\hline
\end{tabular}


Ninth Generation: Variable SD2 is removed from the chromosome since it obtained the lowest fitness value of 277729 as evident in Table 10.

Table 10. G9 using an average crossover with RWS function

\begin{tabular}{ccccccccc}
\hline \multirow{2}{*}{ IP } & \multirow{2}{*}{ X } & \multirow{2}{*}{ Fitness } & \multirow{2}{*}{ Rank } & Y & IP & Off-spring & Fitness & \multirow{2}{*}{ Decision } \\
\hline M1 & 546 & 298116 & 22 & 552 & M5 & 549 & 301401 & \\
M2 & 565 & 319225 & 30 & 565 & M2 & 565 & 319225 & \\
M3 & 558 & 311364 & 27 & 548 & SD1 & 553 & 305809 & \\
M4 & 559 & 312481 & 28 & 546 & SD5 & 552.5 & 305256.3 & \\
C5 & 528 & 278784 & 12 & 531 & ST3 & 529.5 & 280370.3 & \\
SD2 & 512 & 262144 & 5 & 542 & C4 & 527 & 277729 & Remove \\
SD3 & 565 & 319225 & 29 & 546 & A3 & 555.5 & 308580.3 & \\
SD4 & 556 & 309136 & 26 & 558 & M3 & 557 & 310249 & \\
SD5 & 546 & 298116 & 21 & 528 & C5 & 537 & 288369 & \\
A3 & 546 & 298116 & 20 & 513 & A1 & 529.5 & 280370.3 & \\
A4 & 518 & 268324 & 8 & 565 & M2 & 541.5 & 293222.3 & \\
ST1 & 556 & 309136 & 25 & 556 & ST1 & 556 & 309136 & \\
ST2 & 534 & 285156 & 16 & 559 & M4 & 546.5 & 298662.3 & \\
ST3 & 531 & 281961 & 14 & 546 & M1 & 538.5 & 289982.3 & \\
ST4 & 541 & 292681 & 17 & 556 & SD4 & 548.5 & 300852.3 & \\
ST5 & 527 & 277729 & 11 & 552 & M5 & 539.5 & 291060.3 & \\
P1 & 531 & 281961 & 13 & 565 & SD3 & 548 & 300304 & \\
P2 & 533 & 284089 & 15 & 541 & ST4 & 537 & 288369 & \\
P4 & 526 & 276676 & 9 & 565 & SD3 & 545.5 & 297570.3 & \\
P5 & 544 & 295936 & 19 & 565 & SD3 & 554.5 & 307470.3 &
\end{tabular}

Tenth Generation: Variables C5 and A3 were removed from the chromosome since it obtained the lowest fitness value of 280370.3 as evident in Table 11.

Table 11. G10 using an average crossover with RWS function

\begin{tabular}{ccccccccc}
\hline \multirow{2}{*}{ IP } & \multirow{2}{*}{ X } & \multirow{2}{*}{ Fitness } & Rank & Y & IP & Off-spring & \multirow{2}{*}{ Fitness } & \multirow{2}{*}{ Decision } \\
\hline M1 & 546 & 298116 & 22 & 552 & M5 & 549 & 301401 & \\
M2 & 565 & 319225 & 30 & 565 & M2 & 565 & 319225 & \\
M3 & 558 & 311364 & 27 & 548 & SD1 & 553 & 305809 & \\
M4 & 559 & 312481 & 28 & 546 & SD5 & 552.5 & 305256.3 & \\
C5 & 528 & 278784 & 12 & 531 & ST3 & 529.5 & 280370.3 & Remove \\
SD3 & 565 & 319225 & 29 & 546 & A3 & 555.5 & 308580.3 & \\
SD4 & 556 & 309136 & 26 & 558 & M3 & 557 & 310249 & \\
SD5 & 546 & 298116 & 21 & 528 & C5 & 537 & 288369 & \\
A3 & 546 & 298116 & 20 & 513 & A1 & 529.5 & 280370.3 & Remove \\
A4 & 518 & 268324 & 8 & 565 & M2 & 541.5 & 293222.3 & \\
ST1 & 556 & 309136 & 25 & 556 & ST1 & 556 & 309136 & \\
ST2 & 534 & 285156 & 16 & 559 & M4 & 546.5 & 298662.3 & \\
ST3 & 531 & 281961 & 14 & 546 & M1 & 538.5 & 289982.3 & \\
ST4 & 541 & 292681 & 17 & 556 & SD4 & 548.5 & 300852.3 & \\
ST5 & 527 & 277729 & 11 & 552 & M5 & 539.5 & 291060.3 & \\
P1 & 531 & 281961 & 13 & 565 & SD3 & 548 & 300304 & \\
P2 & 533 & 284089 & 15 & 541 & ST4 & 537 & 288369 & \\
P4 & 526 & 276676 & 9 & 565 & SD3 & 545.5 & 297570.3 & \\
P5 & 544 & 295936 & 19 & 565 & SD3 & 554.5 & 307470.3 & \\
\hline
\end{tabular}

\subsection{Simulation result for GA with IBAX operator and rank-based selection function}

The simulation on the genetic algorithm was done utilizing the novel Inversed Bi-segmented Average Crossover (IBAX) operator and rank-based selection function on the same datasets and number of generations.

First Generation: Variable C2 was removed from the list of variables after applying the rank-based selection. The variable $\mathrm{C} 2$ obtained the lowest fitness value in the rank-based selection. Hence, it does not have any chance to be selected. Moreover, after applying the inversed bi-segmented average crossover (IBAX) operator and obtained the fitness value of the offspring, variable $\mathrm{C} 3$ was removed from the chromosomes since it obtained the lowest fitness value of 224676 that will not warrant for the next generation. Thus, in the first generation, there were two variables removed from the list as shown in Table 12. 
Table 12. G1 using IBAX with the rank-based selection function

\begin{tabular}{|c|c|c|c|c|c|c|c|c|}
\hline \multicolumn{5}{|c|}{ Rank-based } & \multicolumn{4}{|c|}{ IBAX } \\
\hline IP & $\mathrm{X}$ & Fitness & Rank & New Fitness & Parent 1 & Parent 2 & Offspring & Fitness \\
\hline M2 & 565 & 319225 & 30 & 3629986.8 & 565 & 541 & 553 & 305809 \\
\hline SD3 & 565 & 319225 & 29 & 3504670.8 & 565 & 542 & 553.5 & 306362.3 \\
\hline M4 & 559 & 312481 & 28 & 3379354.8 & 559 & 544 & 551.5 & 304152.3 \\
\hline M3 & 558 & 311364 & 27 & 3254038.8 & 558 & 546 & 552 & 304704 \\
\hline SD4 & 556 & 309136 & 26 & 3128722.8 & 556 & 546 & 551 & 303601 \\
\hline ST1 & 556 & 309136 & 25 & 3003406.8 & 556 & 546 & 551 & 303601 \\
\hline M5 & 552 & 304704 & 24 & 2878090.8 & 552 & 548 & 550 & 302500 \\
\hline SD1 & 548 & 300304 & 23 & 2752774.8 & 548 & 552 & 550 & 302500 \\
\hline M1 & 546 & 298116 & 22 & 2627458.8 & 546 & 556 & 551 & 303601 \\
\hline SD5 & 546 & 298116 & 21 & 2502142.8 & 546 & 556 & 551 & 303601 \\
\hline A3 & 546 & 298116 & 20 & 2376826.8 & 546 & 558 & 552 & 304704 \\
\hline P5 & 544 & 295936 & 19 & 2251510.8 & 544 & 559 & 551.5 & 304152.3 \\
\hline $\mathrm{C} 4$ & 542 & 293764 & 18 & 2126194.8 & 542 & 565 & 553.5 & 306362.3 \\
\hline ST4 & 541 & 292681 & 17 & 2000878.8 & 541 & 565 & 553 & 305809 \\
\hline ST2 & 534 & 285156 & 16 & 1875562.8 & 534 & 490 & 512 & 262144 \\
\hline $\mathrm{P} 2$ & 533 & 284089 & 15 & 1750246.8 & 533 & 500 & 516.5 & 266772.3 \\
\hline ST3 & 531 & 281961 & 14 & 1624930.8 & 531 & 512 & 521.5 & 271962.3 \\
\hline $\mathrm{P} 1$ & 531 & 281961 & 13 & 1499614.8 & 531 & 513 & 522 & 272484 \\
\hline $\mathrm{C} 5$ & 528 & 278784 & 12 & 1374298.8 & 528 & 516 & 522 & 272484 \\
\hline ST5 & 527 & 277729 & 11 & 1248982.8 & 527 & 518 & 522.5 & 273006.3 \\
\hline P3 & 526 & 276676 & 10 & 1123666.8 & 526 & 526 & 526 & 276676 \\
\hline P4 & 526 & 276676 & 9 & 998350.8 & 526 & 526 & 526 & 276676 \\
\hline $\mathrm{A} 4$ & 518 & 268324 & 8 & 873034.8 & 518 & 527 & 522.5 & 273006.3 \\
\hline A5 & 516 & 266256 & 7 & 747718.8 & 516 & 528 & 522 & 272484 \\
\hline A1 & 513 & 263169 & 6 & 622402.8 & 513 & 531 & 522 & 272484 \\
\hline SD2 & 512 & 262144 & 5 & 497086.8 & 512 & 531 & 521.5 & 271962.3 \\
\hline $\mathrm{A} 2$ & 500 & 250000 & 4 & 371770.8 & 500 & 533 & 516.5 & 266772.3 \\
\hline $\mathrm{C} 1$ & 490 & 240100 & 3 & 246454.8 & 490 & 534 & 512 & 262144 \\
\hline $\mathrm{C} 3$ & 474 & 224676 & 2 & 121138.8 & 474 & 474 & 474 & 224676 \\
\hline $\mathrm{C} 2$ & 354 & 125316 & 1 & -4177.2 & & & & \\
\hline
\end{tabular}

Second Generation: After applying the inversed bi-segmented average crossover (IBAX) operator, variables ST2 and C1 were removed from the chromosomes since they obtained the lowest fitness value of 262144. In the second generation, there were two variables removed from the list as shown in Table 13.

Table 13. G2 using IBAX with the rank-based selection function

\begin{tabular}{cccccccccc}
\hline & \multicolumn{4}{c}{ Rank-based } & & & \multicolumn{3}{c}{ IBAX } \\
IP & $\mathrm{X}$ & Fitness & Rank & New Fitness & & Parent 1 & Parent 2 & Offspring & Fitness \\
\hline M2 & 565 & 319225 & 30 & 3629986.8 & & 565 & 541 & 553 & 305809 \\
SD3 & 565 & 319225 & 29 & 3504670.8 & & 565 & 542 & 553.5 & 306362.3 \\
M4 & 559 & 312481 & 28 & 3379354.8 & & 559 & 544 & 551.5 & 304152.3 \\
M3 & 558 & 311364 & 27 & 3254038.8 & & 558 & 546 & 552 & 304704 \\
SD4 & 556 & 309136 & 26 & 3128722.8 & & 556 & 546 & 551 & 303601 \\
ST1 & 556 & 309136 & 25 & 3003406.8 & & 556 & 546 & 551 & 303601 \\
M5 & 552 & 304704 & 24 & 2878090.8 & & 552 & 548 & 550 & 302500 \\
SD1 & 548 & 300304 & 23 & 2752774.8 & & 548 & 552 & 550 & 302500 \\
M1 & 546 & 298116 & 22 & 2627458.8 & & 546 & 556 & 551 & 303601 \\
SD5 & 546 & 298116 & 21 & 2502142.8 & & 546 & 556 & 551 & 303601 \\
A3 & 546 & 298116 & 20 & 2376826.8 & & 546 & 558 & 552 & 304704 \\
P5 & 544 & 295936 & 19 & 2251510.8 & & 544 & 559 & 551.5 & 304152.3 \\
C4 & 542 & 293764 & 18 & 2126194.8 & & 542 & 565 & 553.5 & 306362.3 \\
ST4 & 541 & 292681 & 17 & 2000878.8 & & 541 & 565 & 553 & 305809 \\
ST2 & 534 & 285156 & 16 & 1875562.8 & & 534 & 490 & 512 & 262144 \\
P2 & 533 & 284089 & 15 & 1750246.8 & & 533 & 500 & 516.5 & 266772.3 \\
ST3 & 531 & 281961 & 14 & 1624930.8 & & 531 & 512 & 521.5 & 271962.3 \\
P1 & 531 & 281961 & 13 & 1499614.8 & & 531 & 513 & 522 & 272484 \\
C5 & 528 & 278784 & 12 & 1374298.8 & & 528 & 516 & 522 & 272484 \\
ST5 & 527 & 277729 & 11 & 1248982.8 & & 527 & 518 & 522.5 & 273006.3 \\
P3 & 526 & 276676 & 10 & 1123666.8 & & 526 & 526 & 526 & 276676 \\
P4 & 526 & 276676 & 9 & 998350.8 & & 526 & 526 & 526 & 276676 \\
A4 & 518 & 268324 & 8 & 873034.8 & & 518 & 527 & 522.5 & 273006.3 \\
A5 & 516 & 266256 & 7 & 747718.8 & & 516 & 528 & 522 & 272484 \\
A1 & 513 & 263169 & 6 & 622402.8 & & 513 & 531 & 522 & 272484 \\
SD2 & 512 & 262144 & 5 & 497086.8 & & 512 & 531 & 521.5 & 271962.3 \\
A2 & 500 & 250000 & 4 & 371770.8 & & 500 & 533 & 516.5 & 266772.3 \\
C1 & 490 & 240100 & 3 & 246454.8 & & 490 & 534 & 512 & 262144 \\
\hline & & & & & & & & &
\end{tabular}


Third Generation: After applying the inversed bi-segmented average crossover (IBAX) operator and obtained the fitness value of the offspring, variables ST4 and A2 were removed from the chromosomes since both obtained the lowest fitness value of 270920.3. In the third generation, there were two variables removed from the list as shown in Table 14.

Table 14. G3 using IBAX with the rank-based selection function

\begin{tabular}{|c|c|c|c|c|c|c|c|c|}
\hline \multicolumn{5}{|c|}{ Rank-based } & \multicolumn{4}{|c|}{ IBAX } \\
\hline IP & $X$ & Fitness & Rank & New Fitness & Parent 1 & Parent 2 & Offspring & Fitness \\
\hline M2 & 565 & 319225 & 30 & 3629986.8 & 565 & 542 & 553.5 & 306362.3 \\
\hline SD3 & 565 & 319225 & 29 & 3504670.8 & 565 & 544 & 554.5 & 307470.3 \\
\hline M4 & 559 & 312481 & 28 & 3379354.8 & 559 & 546 & 552.5 & 305256.3 \\
\hline M3 & 558 & 311364 & 27 & 3254038.8 & 558 & 546 & 552 & 304704 \\
\hline SD4 & 556 & 309136 & 26 & 3128722.8 & 556 & 546 & 551 & 303601 \\
\hline ST1 & 556 & 309136 & 25 & 3003406.8 & 556 & 548 & 552 & 304704 \\
\hline M5 & 552 & 304704 & 24 & 2878090.8 & 552 & 552 & 552 & 304704 \\
\hline SD1 & 548 & 300304 & 23 & 2752774.8 & 548 & 556 & 552 & 304704 \\
\hline M1 & 546 & 298116 & 22 & 2627458.8 & 546 & 556 & 551 & 303601 \\
\hline SD5 & 546 & 298116 & 21 & 2502142.8 & 546 & 558 & 552 & 304704 \\
\hline A3 & 546 & 298116 & 20 & 2376826.8 & 546 & 559 & 552.5 & 305256.3 \\
\hline P5 & 544 & 295936 & 19 & 2251510.8 & 544 & 565 & 554.5 & 307470.3 \\
\hline $\mathrm{C} 4$ & 542 & 293764 & 18 & 2126194.8 & 542 & 565 & 553.5 & 306362.3 \\
\hline ST4 & 541 & 292681 & 17 & 2000878.8 & 541 & 500 & 520.5 & 270920.3 \\
\hline $\mathrm{P} 2$ & 533 & 284089 & 15 & 1750246.8 & 533 & 512 & 522.5 & 273006.3 \\
\hline ST3 & 531 & 281961 & 14 & 1624930.8 & 531 & 513 & 522 & 272484 \\
\hline P1 & 531 & 281961 & 13 & 1499614.8 & 531 & 516 & 523.5 & 274052.3 \\
\hline $\mathrm{C} 5$ & 528 & 278784 & 12 & 1374298.8 & 528 & 518 & 523 & 273529 \\
\hline ST5 & 527 & 277729 & 11 & 1248982.8 & 527 & 526 & 526.5 & 277202.3 \\
\hline P3 & 526 & 276676 & 10 & 1123666.8 & 526 & 526 & 526 & 276676 \\
\hline $\mathrm{P} 4$ & 526 & 276676 & 9 & 998350.8 & 526 & 527 & 526.5 & 277202.3 \\
\hline A4 & 518 & 268324 & 8 & 873034.8 & 518 & 528 & 523 & 273529 \\
\hline A5 & 516 & 266256 & 7 & 747718.8 & 516 & 531 & 523.5 & 274052.3 \\
\hline A1 & 513 & 263169 & 6 & 622402.8 & 513 & 531 & 522 & 272484 \\
\hline SD2 & 512 & 262144 & 5 & 497086.8 & 512 & 533 & 522.5 & 273006.3 \\
\hline $\mathrm{A} 2$ & 500 & 250000 & 4 & 371770.8 & 500 & 541 & 520.5 & 270920.3 \\
\hline
\end{tabular}

Fourth Generation: After applying the inversed bi-segmented average crossover (IBAX) operator, variables P2 and A1 were removed from the chromosomes since both obtained the lowest fitness value of 273529. In the fourth generation, there were two variables removed from the list as shown in Table 15.

Table 15. G4 using IBAX with the rank-based selection function

\begin{tabular}{|c|c|c|c|c|c|c|c|c|}
\hline \multicolumn{5}{|c|}{ Rank-based } & \multicolumn{4}{|c|}{ IBAX } \\
\hline IP & $\mathrm{X}$ & Fitness & Rank & New Fitness & Parent 1 & Parent 2 & Offspring & Fitness \\
\hline M2 & 565 & 319225 & 30 & 3629986.8 & 565 & 544 & 554.5 & 307470.3 \\
\hline SD3 & 565 & 319225 & 29 & 3504670.8 & 565 & 546 & 555.5 & 308580.3 \\
\hline M4 & 559 & 312481 & 28 & 3379354.8 & 559 & 546 & 552.5 & 305256.3 \\
\hline M3 & 558 & 311364 & 27 & 3254038.8 & 558 & 546 & 552 & 304704 \\
\hline SD4 & 556 & 309136 & 26 & 3128722.8 & 556 & 548 & 552 & 304704 \\
\hline ST1 & 556 & 309136 & 25 & 3003406.8 & 556 & 552 & 554 & 306916 \\
\hline M5 & 552 & 304704 & 24 & 2878090.8 & 552 & 556 & 554 & 306916 \\
\hline SD1 & 548 & 300304 & 23 & 2752774.8 & 548 & 556 & 552 & 304704 \\
\hline M1 & 546 & 298116 & 22 & 2627458.8 & 546 & 558 & 552 & 304704 \\
\hline SD5 & 546 & 298116 & 21 & 2502142.8 & 546 & 559 & 552.5 & 305256.3 \\
\hline A3 & 546 & 298116 & 20 & 2376826.8 & 546 & 565 & 555.5 & 308580.3 \\
\hline P5 & 544 & 295936 & 19 & 2251510.8 & 544 & 565 & 554.5 & 307470.3 \\
\hline $\mathrm{C} 4$ & 542 & 293764 & 18 & 2126194.8 & 542 & 512 & 527 & 277729 \\
\hline $\mathrm{P} 2$ & 533 & 284089 & 15 & 1750246.8 & 533 & 513 & 523 & 273529 \\
\hline ST3 & 531 & 281961 & 14 & 1624930.8 & 531 & 516 & 523.5 & 274052.3 \\
\hline $\mathrm{P} 1$ & 531 & 281961 & 13 & 1499614.8 & 531 & 518 & 524.5 & 275100.3 \\
\hline $\mathrm{C} 5$ & 528 & 278784 & 12 & 1374298.8 & 528 & 526 & 527 & 277729 \\
\hline ST5 & 527 & 277729 & 11 & 1248982.8 & 527 & 526 & 526.5 & 277202.3 \\
\hline P3 & 526 & 276676 & 10 & 1123666.8 & 526 & 527 & 526.5 & 277202.3 \\
\hline $\mathrm{P} 4$ & 526 & 276676 & 9 & 998350.8 & 526 & 528 & 527 & 277729 \\
\hline A4 & 518 & 268324 & 8 & 873034.8 & 518 & 531 & 524.5 & 275100.3 \\
\hline A5 & 516 & 266256 & 7 & 747718.8 & 516 & 531 & 523.5 & 274052.3 \\
\hline A1 & 513 & 263169 & 6 & 622402.8 & 513 & 533 & 523 & 273529 \\
\hline SD2 & 512 & 262144 & 5 & 497086.8 & 512 & 542 & 527 & 277729 \\
\hline
\end{tabular}


Fifth Generation: After applying the inversed bi-segmented average crossover (IBAX) operator, variables ST3 and A4 were removed from the chromosomes since both obtained the lowest fitness value of 275100.3. In the fifth generation, there were two variables removed from the list as shown in Table 16.

Table 16. G5 using IBAX with the rank-based selection function

\begin{tabular}{ccccc}
\hline \multicolumn{5}{c}{ Rank-based } \\
IP & X & Fitness & Rank & New Fitness \\
\hline M2 & 565 & 319225 & 30 & 3629986.8 \\
SD3 & 565 & 319225 & 29 & 3504670.8 \\
M4 & 559 & 312481 & 28 & 3379354.8 \\
M3 & 558 & 311364 & 27 & 3254038.8 \\
SD4 & 556 & 309136 & 26 & 3128722.8 \\
ST1 & 556 & 309136 & 25 & 3003406.8 \\
M5 & 552 & 304704 & 24 & 2878090.8 \\
SD1 & 548 & 300304 & 23 & 2752774.8 \\
M1 & 546 & 298116 & 22 & 2627458.8 \\
SD5 & 546 & 298116 & 21 & 2502142.8 \\
A3 & 546 & 298116 & 20 & 2376826.8 \\
P5 & 544 & 295936 & 19 & 2251510.8 \\
C4 & 542 & 293764 & 18 & 2126194.8 \\
ST3 & 531 & 281961 & 14 & 1624930.8 \\
P1 & 531 & 281961 & 13 & 1499614.8 \\
C5 & 528 & 278784 & 12 & 1374298.8 \\
ST5 & 527 & 277729 & 11 & 1248982.8 \\
P3 & 526 & 276676 & 10 & 1123666.8 \\
P4 & 526 & 276676 & 9 & 998350.8 \\
A4 & 518 & 268324 & 8 & 873034.8 \\
A5 & 516 & 266256 & 7 & 747718.8 \\
SD2 & 512 & 262144 & 5 & 497086.8 \\
\hline & & & &
\end{tabular}

\begin{tabular}{cccc} 
Parent 1 & Parent 2 & Offspring & Fitness \\
\hline 565 & 546 & 555.5 & 308580.3 \\
565 & 546 & 555.5 & 308580.3 \\
559 & 546 & 552.5 & 305256.3 \\
558 & 548 & 553 & 305809 \\
556 & 552 & 554 & 306916 \\
556 & 556 & 556 & 309136 \\
552 & 556 & 554 & 306916 \\
548 & 558 & 553 & 305809 \\
546 & 559 & 552.5 & 305256.3 \\
546 & 565 & 555.5 & 308580.3 \\
546 & 565 & 555.5 & 308580.3 \\
544 & 512 & 528 & 278784 \\
542 & 516 & 529 & 279841 \\
531 & 518 & 524.5 & 275100.3 \\
531 & 526 & 528.5 & 279312.3 \\
528 & 526 & 527 & 277729 \\
527 & 527 & 527 & 277729 \\
526 & 528 & 527 & 277729 \\
526 & 531 & 528.5 & 279312.3 \\
518 & 531 & 524.5 & 275100.3 \\
516 & 542 & 529 & 279841 \\
512 & 544 & 528 & 278784 \\
\hline
\end{tabular}

Sixth Generation: After applying the inversed bi-segmented average crossover (IBAX) operator, variables C5 and ST5 were removed from the chromosomes since both obtained the lowest fitness value of 278256.3. In the sixth generation, there were two variables removed from the list as shown in Table 17.

Table 17. G6 using IBAX with the rank-based selection function

\begin{tabular}{ccccc}
\hline \multicolumn{4}{c}{ Rank-based } \\
IP & $X$ & Fitness & Rank & New Fitness \\
\hline M2 & 565 & 319225 & 30 & 3629986.8 \\
SD3 & 565 & 319225 & 29 & 3504670.8 \\
M4 & 559 & 312481 & 28 & 3379354.8 \\
M3 & 558 & 311364 & 27 & 3254038.8 \\
SD4 & 556 & 309136 & 26 & 3128722.8 \\
ST1 & 556 & 309136 & 25 & 3003406.8 \\
M5 & 552 & 304704 & 24 & 2878090.8 \\
SD1 & 548 & 300304 & 23 & 2752774.8 \\
M1 & 546 & 298116 & 22 & 2627458.8 \\
SD5 & 546 & 298116 & 21 & 2502142.8 \\
A3 & 546 & 298116 & 20 & 2376826.8 \\
P5 & 544 & 295936 & 19 & 2251510.8 \\
C4 & 542 & 293764 & 18 & 2126194.8 \\
P1 & 531 & 281961 & 13 & 1499614.8 \\
C5 & 528 & 278784 & 12 & 1374298.8 \\
ST5 & 527 & 277729 & 11 & 1248982.8 \\
P3 & 526 & 276676 & 10 & 1123666.8 \\
P4 & 526 & 276676 & 9 & 998350.8 \\
A5 & 516 & 266256 & 7 & 747718.8 \\
SD2 & 512 & 262144 & 5 & 497086.8 \\
\hline
\end{tabular}

\begin{tabular}{|c|c|c|c|}
\hline \multicolumn{4}{|c|}{ IBAX } \\
\hline Parent & Parent 2 & Offspring & Fitness \\
\hline 565 & 546 & 555.5 & 308580.3 \\
\hline 565 & 546 & 555.5 & 308580.3 \\
\hline 559 & 548 & 553.5 & 306362.3 \\
\hline 558 & 552 & 555 & 308025 \\
\hline 556 & 556 & 556 & 309136 \\
\hline 556 & 556 & 556 & 309136 \\
\hline 552 & 558 & 555 & 308025 \\
\hline 548 & 559 & 553.5 & 306362.3 \\
\hline 546 & 565 & 555.5 & 308580.3 \\
\hline 546 & 565 & 555.5 & 308580.3 \\
\hline 546 & 512 & 529 & 279841 \\
\hline 544 & 516 & 530 & 280900 \\
\hline 542 & 526 & 534 & 285156 \\
\hline 531 & 526 & 528.5 & 279312.3 \\
\hline 528 & 527 & 527.5 & 278256.3 \\
\hline 527 & 528 & 527.5 & 278256.3 \\
\hline 526 & 531 & 528.5 & 279312.3 \\
\hline 526 & 542 & 534 & 285156 \\
\hline 516 & 544 & 530 & 280900 \\
\hline 512 & 546 & 529 & 279841 \\
\hline
\end{tabular}

Seventh Generation: After applying the inversed bi-segmented average crossover (IBAX) operator, variables SD5 and SD2 were removed from the chromosomes since both obtained the lowest fitness value of 279841. In the seventh generation, there were two variables removed from the list as shown in Table 18. 
Table 18. G7 using IBAX with the rank-based selection function

\begin{tabular}{cccccccccc}
\hline & \multicolumn{4}{c}{ Rank-based } & & & \multicolumn{3}{c}{ IBAX } \\
IP & $\mathrm{X}$ & Fitness & Rank & New Fitness & & Parent 1 & Parent 2 & Offspring & Fitness \\
\hline M2 & 565 & 319225 & 30 & 3629986.8 & & 565 & 546 & 555.5 & 308580.3 \\
SD3 & 565 & 319225 & 29 & 3504670.8 & & 565 & 548 & 556.5 & 309692.3 \\
M4 & 559 & 312481 & 28 & 3379354.8 & & 559 & 552 & 555.5 & 308580.3 \\
M3 & 558 & 311364 & 27 & 3254038.8 & & 558 & 556 & 557 & 310249 \\
SD4 & 556 & 309136 & 26 & 3128722.8 & & 556 & 556 & 556 & 309136 \\
ST1 & 556 & 309136 & 25 & 3003406.8 & & 556 & 558 & 557 & 310249 \\
M5 & 552 & 304704 & 24 & 2878090.8 & & 552 & 559 & 555.5 & 308580.3 \\
SD1 & 548 & 300304 & 23 & 2752774.8 & & 548 & 565 & 556.5 & 309692.3 \\
M1 & 546 & 298116 & 22 & 2627458.8 & & 546 & 565 & 555.5 & 308580.3 \\
SD5 & 546 & 298116 & 21 & 2502142.8 & & 546 & 512 & 529 & 279841 \\
A3 & 546 & 298116 & 20 & 2376826.8 & & 546 & 516 & 531 & 281961 \\
P5 & 544 & 295936 & 19 & 2251510.8 & & 544 & 526 & 535 & 286225 \\
C4 & 542 & 293764 & 18 & 2126194.8 & & 542 & 526 & 534 & 285156 \\
P1 & 531 & 281961 & 13 & 1499614.8 & & 531 & 531 & 531 & 281961 \\
P3 & 526 & 276676 & 10 & 1123666.8 & & 526 & 542 & 534 & 285156 \\
P4 & 526 & 276676 & 9 & 998350.8 & & 526 & 544 & 535 & 286225 \\
A5 & 516 & 266256 & 7 & 747718.8 & & 516 & 546 & 531 & 281961 \\
SD2 & 512 & 262144 & 5 & 497086.8 & & 512 & 546 & 529 & 279841 \\
\hline
\end{tabular}

Eighth Generation: After applying the inversed bi-segmented average crossover (IBAX) operator, variables M1 and A5 were removed from the chromosomes since both obtained the lowest fitness value of 281961. In the eight generations, there were two variables removed from the list as shown in Table 19.

Ninth Generation: After applying the inversed bi-segmented average crossover (IBAX) operator, variables A3 and P3 were removed from the chromosomes since both obtained the lowest fitness value of 287296. In the ninth generation, there were two variables removed from the list as shown in Table 20.

Table 19. G8 using IBAX with rank-based selection function

\begin{tabular}{ccccc}
\hline & \multicolumn{4}{c}{ Rank-based } \\
IP & $X$ & Fitness & Rank & New Fitness \\
\hline M2 & 565 & 319225 & 30 & 3629986.8 \\
SD3 & 565 & 319225 & 29 & 3504670.8 \\
M4 & 559 & 312481 & 28 & 3379354.8 \\
M3 & 558 & 311364 & 27 & 3254038.8 \\
SD4 & 556 & 309136 & 26 & 3128722.8 \\
ST1 & 556 & 309136 & 25 & 3003406.8 \\
M5 & 552 & 304704 & 24 & 2878090.8 \\
SD1 & 548 & 300304 & 23 & 2752774.8 \\
M1 & 546 & 298116 & 22 & 2627458.8 \\
A3 & 546 & 298116 & 20 & 2376826.8 \\
P5 & 544 & 295936 & 19 & 2251510.8 \\
C4 & 542 & 293764 & 18 & 2126194.8 \\
P1 & 531 & 281961 & 13 & 1499614.8 \\
P3 & 526 & 276676 & 10 & 1123666.8 \\
P4 & 526 & 276676 & 9 & 998350.8 \\
A5 & 516 & 266256 & 7 & 747718.8 \\
\hline
\end{tabular}

\begin{tabular}{cccc}
\hline \multicolumn{4}{c}{ IBAX } \\
Parent 1 & Parent 2 & Offspring & Fitness \\
\hline 565 & 548 & 556.5 & 309692.3 \\
565 & 552 & 558.5 & 311922.3 \\
559 & 556 & 557.5 & 310806.3 \\
558 & 556 & 557 & 310249 \\
556 & 558 & 557 & 310249 \\
556 & 559 & 557.5 & 310806.3 \\
552 & 565 & 558.5 & 311922.3 \\
548 & 565 & 556.5 & 309692.3 \\
546 & 516 & 531 & 281961 \\
546 & 526 & 536 & 287296 \\
544 & 526 & 535 & 286225 \\
542 & 531 & 536.5 & 287832.3 \\
531 & 542 & 536.5 & 287832.3 \\
526 & 544 & 535 & 286225 \\
526 & 546 & 536 & 287296 \\
516 & 546 & 531 & 281961 \\
\hline
\end{tabular}

Table 20. G9 using IBAX with the rank-based selection function

\begin{tabular}{ccccc}
\hline \multicolumn{4}{c}{ Rank-based } \\
IP & X & Fitness & Rank & New Fitness \\
\hline M2 & 565 & 319225 & 30 & 3629986.8 \\
SD3 & 565 & 319225 & 29 & 3504670.8 \\
M4 & 559 & 312481 & 28 & 3379354.8 \\
M3 & 558 & 311364 & 27 & 3254038.8 \\
SD4 & 556 & 309136 & 26 & 3128722.8 \\
ST1 & 556 & 309136 & 25 & 3003406.8 \\
M5 & 552 & 304704 & 24 & 2878090.8 \\
SD1 & 548 & 300304 & 23 & 2752774.8 \\
A3 & 546 & 298116 & 20 & 2376826.8 \\
P5 & 544 & 295936 & 19 & 2251510.8 \\
C4 & 542 & 293764 & 18 & 2126194.8 \\
P1 & 531 & 281961 & 13 & 1499614.8 \\
P3 & 526 & 276676 & 10 & 1123666.8 \\
P4 & 526 & 276676 & 9 & 998350.8 \\
\hline
\end{tabular}

\begin{tabular}{cccc}
\hline \multicolumn{4}{c}{ IBAX } \\
Parent 1 & Parent 2 & Offspring & Fitness \\
\hline 565 & 552 & 558.5 & 311922.3 \\
565 & 556 & 560.5 & 314160.3 \\
559 & 556 & 557.5 & 310806.3 \\
558 & 558 & 558 & 311364 \\
556 & 559 & 557.5 & 310806.3 \\
556 & 565 & 560.5 & 314160.3 \\
552 & 565 & 558.5 & 311922.3 \\
548 & 526 & 537 & 288369 \\
546 & 526 & 536 & 287296 \\
544 & 531 & 537.5 & 288906.3 \\
542 & 542 & 542 & 293764 \\
531 & 544 & 537.5 & 288906.3 \\
526 & 546 & 536 & 287296 \\
526 & 548 & 537 & 288369 \\
\hline
\end{tabular}


Tenth Generation: After applying the inversed bi-segmented average crossover (IBAX) operator, variables M5 and P4 were removed from the chromosomes since both obtained the lowest fitness value of 290521. In the tenth generation, there were two variables removed from the list as shown in Table 21.

Table 21. G10 using IBAX with the rank-based selection function

\begin{tabular}{ccccc}
\hline \multicolumn{5}{c}{ Rank-based } \\
IP & $X$ & Fitness & Rank & New Fitness \\
\hline M2 & 565 & 319225 & 30 & 3629986.8 \\
SD3 & 565 & 319225 & 29 & 3504670.8 \\
M4 & 559 & 312481 & 28 & 3379354.8 \\
M3 & 558 & 311364 & 27 & 3254038.8 \\
SD4 & 556 & 309136 & 26 & 3128722.8 \\
ST1 & 556 & 309136 & 25 & 3003406.8 \\
M5 & 552 & 304704 & 24 & 2878090.8 \\
SD1 & 548 & 300304 & 23 & 2752774.8 \\
P5 & 544 & 295936 & 19 & 2251510.8 \\
C4 & 542 & 293764 & 18 & 2126194.8 \\
P1 & 531 & 281961 & 13 & 1499614.8 \\
P4 & 526 & 276676 & 9 & 998350.8 \\
\hline
\end{tabular}

\begin{tabular}{cccc}
\hline \multicolumn{4}{c}{ IBAX } \\
Parent 1 & Parent 2 & Offspring & Fitness \\
\hline 565 & 556 & 560.5 & 314160.3 \\
565 & 556 & 560.5 & 314160.3 \\
559 & 558 & 558.5 & 311922.3 \\
558 & 559 & 558.5 & 311922.3 \\
556 & 565 & 560.5 & 314160.3 \\
556 & 565 & 560.5 & 314160.3 \\
552 & 526 & 539 & 290521 \\
548 & 531 & 539.5 & 291060.3 \\
544 & 542 & 543 & 294849 \\
542 & 544 & 543 & 294849 \\
531 & 548 & 539.5 & 291060.3 \\
526 & 552 & 539 & 290521 \\
\hline
\end{tabular}

\subsection{Evaluation of the efficacy and reduction rate using GA with $\mathrm{AX}$ and IBAX operators}

The variable minimization process using the genetic algorithm with average crossover operator and roulette wheel selection function has depicted a decrease after the ten generations. From the 30 variables, the numbers were minimized to 17 variables. $43 \%$ of variables were removed as depicted in Table 22 .

Meanwhile, the variable minimization process using the genetic algorithm with the proposed novel mating scheme called inversed bi-segmented average crossover operator, and rank-based selection function has depicted a noticeable decrease after the ten generations. From the 30 variables, the numbers were minimized to 10 variables after the generations. A total of $66.66 \%$ of variables were removed as depicted in Table 23. Since the amount of reduction varies according to the genetic algorithms used, removing $66.66 \%$ of the variables in the dataset is good enough as the notion of dropping one or more variables should help reduce dimensionality is certain. The ratio of feature reduction of more than $60 \%$ is acceptable just like in the work of [31].

Table 22. Simulation result for GA with AX operator and RWS function

\begin{tabular}{ccccc}
\hline $\begin{array}{c}\text { Number of } \\
\text { Generations }\end{array}$ & $\begin{array}{c}\text { Number of } \\
\text { Variables Left }\end{array}$ & $\begin{array}{c}\text { Number of Variables } \\
\text { Removed }\end{array}$ & $\begin{array}{c}\text { Variables } \\
\text { Removed }\end{array}$ & Percentage \\
\hline 1 & 30 & 1 & $\mathrm{C} 2$ & $3.33 \%$ \\
2 & 29 & 2 & M5, A2 & $6.66 \%$ \\
3 & 27 & 1 & $\mathrm{C} 3$ & $3.33 \%$ \\
4 & 26 & 2 & $\mathrm{C} 4, \mathrm{~A} 5$ & $6.66 \%$ \\
5 & 24 & 1 & $\mathrm{C} 1$ & $3.33 \%$ \\
6 & 23 & 1 & $\mathrm{P} 3$ & $3.33 \%$ \\
7 & 22 & 1 & $\mathrm{~A} 1$ & $3.33 \%$ \\
8 & 21 & 1 & $\mathrm{SD} 1$ & $3.33 \%$ \\
9 & 20 & 1 & $\mathrm{SD} 2$ & $3.33 \%$ \\
10 & 19 & 2 & $\mathrm{C} 5, \mathrm{~A} 3$ & $6.66 \%$ \\
10 & 17 & - & - & - \\
& Total Percentage of Variables Removed & & $43.33 \%$ \\
\hline
\end{tabular}

Table 23. Simulation result for GA with IBAX operator and rank-based selection function

\begin{tabular}{ccccc}
\hline $\begin{array}{c}\text { Number of } \\
\text { Generations }\end{array}$ & $\begin{array}{c}\text { Number of } \\
\text { Variables Left }\end{array}$ & $\begin{array}{c}\text { Number of Variables } \\
\text { Removed }\end{array}$ & $\begin{array}{c}\text { Variables } \\
\text { Removed }\end{array}$ & Percentage \\
\hline 1 & 30 & 2 & $\mathrm{C} 3, \mathrm{C} 2$ & $6.66 \%$ \\
2 & 28 & 2 & $\mathrm{ST} 2, \mathrm{C} 1$ & $6.66 \%$ \\
3 & 26 & 2 & $\mathrm{ST} 4, \mathrm{~A} 2$ & $6.66 \%$ \\
4 & 24 & 2 & $\mathrm{P} 2, \mathrm{~A} 1$ & $6.66 \%$ \\
5 & 22 & 2 & $\mathrm{ST} 3, \mathrm{~A} 4$ & $6.66 \%$ \\
6 & 20 & 2 & $\mathrm{C} 5, \mathrm{ST} 5$ & $6.66 \%$ \\
7 & 18 & 2 & $\mathrm{SD} 5, \mathrm{SD} 2$ & $6.66 \%$ \\
8 & 16 & $\mathrm{M} 1, \mathrm{~A} 5$ & $6.66 \%$ \\
9 & 14 & 2 & $\mathrm{~A} 3, \mathrm{P} 3$ & $6.66 \%$ \\
10 & 12 & 2 & - & $6.66 \%$ \\
10 & 10 & - & & - \\
& Total Percentage of Variables Removed & & $66.66 \%$ \\
\hline
\end{tabular}

A modified genetic algorithm with a new crossover mating scheme (Allemar Jhone P. Delima) 
To have a further evaluation on the efficacy and reduction rate of the proposed novel crossover, the GA with the IBAX operator was compared along with the other real encoding-based crossover mechanism of the GA such as geometrical crossover [32] and cross average crossover (CAX) [22] aside from the AX operator. The simulation result showed that the genetic algorithm with a new crossover mating scheme outperformed the other existing real encoding-based crossover operators of genetic algorithm in reducing variables as depicted in Table 24.

Table 24. Comparative result for variable minimization using genetic algorithms

\begin{tabular}{ccccc}
\hline Genetic Algorithms & $\begin{array}{c}\text { Number of } \\
\text { variables }\end{array}$ & $\begin{array}{c}\text { Number of } \\
\text { variables left }\end{array}$ & $\begin{array}{c}\text { Number of } \\
\text { variables removed }\end{array}$ & $\begin{array}{c}\text { Percentage of } \\
\text { variables removed }\end{array}$ \\
\hline $\begin{array}{c}\text { GA with AX operator } \\
\text { and RWS function }\end{array}$ & 30 & 17 & 13 & $43.33 \%$ \\
$\begin{array}{c}\text { GA with Geometrical } \\
\text { crossover operator } \\
\text { with RWS function }\end{array}$ & 30 & 20 & 10 & $33.33 \%$ \\
$\begin{array}{c}\text { GA with CAX } \\
\text { operator with RWS } \\
\quad \text { function }\end{array}$ & 30 & 13 & 17 & $56.66 \%$ \\
$\begin{array}{c}\text { GA with IBAX operator } \\
\text { and Rank-based } \\
\text { Selection function }\end{array}$ & 30 & 10 & 20 & $66.66 \%$ \\
\hline
\end{tabular}

\section{CONCLUSION AND RECOMMENDATION}

Through the study, a novel approach for the optimization process using another crossover operator of genetic algorithm was introduced and added to the body of knowledge. The proposed modification on the genetic algorithm with inversed bi-segmented average crossover (IBAX) has paved the way to the enhancement of GA's average crossover mating scheme that affects GA's optimization performance in general. It is evident that the IBAX operator performed the minimization process way better than the average crossover since there were 10 and 17 variables left, respectively, after the tenth generations.

For future works, it is suggested to use the novel IBAX operator in the different type of datasets and incorporate the modified genetic algorithm in various data mining techniques and approaches that need variable minimization or feature reduction process such as in prediction.

\section{REFERENCES}

[1] E. S. K. Tiu, Y. F. Huang, and L. Ling, "Improving the performance of streamflow forecasting model using datapreprocessing technique in Dungun River Basin," E3S Web Conf., vol. 34, no. 02014, 2018.

[2] M. Munk and L. Benko, "Using Entropy in Web Usage Data Preprocessing," Entropy, vol. 20, no. 67, pp. 1-15, 2018.

[3] A. Roy, R. M. O. Cruz, R. Sabourin, and G. D. C. Cavalcanti, "A study on combining dynamic selection and data preprocessing for imbalance learning," Neurocomputing, vol. 286, pp. 179-192, 2018.

[4] P. Vrushali, M. Trupti, G. Pratiksha, and G. Arti, "Crime Rate Prediction using KNN," Int. J. Recent Innov. Trends Comput. Commun., vol. 6, no. 1, pp. 124-127, 2018.

[5] S. García, J. Luengo, and F. Herrera, "Tutorial on practical tips of the most influential data preprocessing algorithms in data mining," Knowledge-Based Syst., vol. 98, pp. 1-29, 2016.

[6] A. Baldominos, P. Isasi, and U. C. I. I. I. De Madrid, "Feature Set Optimization for Physical Activity Recognition Using Genetic Algorithms,” Proc. Companion Publ. 2015 Genet. Evol. Comput. Conf. - GECCO Companion '15, pp. 1311-1318, 2015.

[7] C.-F. J. Kuo, C.-H. Lin, and M.-H. Lee, "Analyze the energy consumption characteristics and affecting factors of Taiwan's convenience stores-using the big data mining approach,” Energy Build., vol. 168, pp. 120-136, 2018.

[8] I. M. El-hasnony, H. M. El Bakry, and A. A. Saleh, "Comparative Study among Data Reduction Techniques over Classification Accuracy,” Int. J. Comput. Appl., vol. 122, no. 2, pp. 9-15, 2015.

[9] F. Herrera and S. Garc, "Prototype Selection for Nearest Neighbor Classification Taxonomy and Empirical Study," IEEE Trans. Pattern Anal. Mach. Intell., vol. 34, no. 3, pp. 417-435, 2012.

[10] J. R. Cano, F. Herrera, and M. Lozano, "On the combination of evolutionary algorithms and stratified strategies for training set selection in data mining," Appl. Soft Comput. J., vol. 6, no. 3, pp. 323-332, 2006.

[11] Y. Cheng, K. Chen, H. Sun, Y. Zhang, and F. Tao, "Data and knowledge mining with big data towards smart production," J. Ind. Inf. Integr., vol. 9, pp. 1-13, 2018.

[12] S. Ramírez-Gallego, B. Krawczyk, S. García, M. Woźniak, and F. Herrera, "A survey on data preprocessing for data stream mining: Current status and future directions," Neurocomputing, vol. 239, pp. 39-57, 2017.

[13] M. Mafarja, I. Aljarah, A. A. Heidari, A. I. Hammouri, H. Faris, and A. M. Al-zoubi, "Evolutionary Population Dynamics and Grasshopper Optimization Approaches for Feature Selection Problems," Knowledge-Based Syst., vol. 154, no. 7, pp. 25-45, 2017.

Indo. J. Elec. Eng. \& Inf, Vol.7, No. 2, June 2019: 165 - 181 
[14] S. R. A. Archana and M. S. Thanabal, "Optimization Algorithms for Feature Selection in Classification : A Survey," Int. J. Innov. Res. Comput. Commun. Eng., vol. 4, no. 2, pp. 1124-1127, 2016.

[15] C. Tsai, W. Eberle, and C. Chu, "Knowledge-Based Systems Genetic algorithms in feature and instance selection," Knowledge-Based Syst., vol. 39, pp. 240-24, 2013.

[16] P. Horton, M. Jaboyedoff, and C. Obled, "Using genetic algorithms to optimize the analogue method for precipitation prediction in the Swiss Alps," J. Hydrol., vol. 556, pp. 1220-1231, 2018.

[17] S. F. Galan, O. J. Mengshoel, and R. Pinter, "A novel mating approach for genetic algorithms," Evol. Comput., vol. 21, no. 2, pp. 197-229, 2013.

[18] V. K. Dabhi and S. Chaudhary, "Empirical modeling using genetic programming: a survey of issues and approaches," Nat. Comput., vol. 14, no. 2, pp. 303-330, 2015.

[19] S. M. Lim, A. B. Sultan, N. Sulaiman, A. Mustapha, and K. Y. Leong, "Crossover and Mutation Operators of Genetic Algorithms," Int. J. Mach. Learn. Comput., vol. 7, no. 1, pp. 10-13, 2017.

[20] S. Picek, "Comparison of a Crossover Operator in Binary-coded Genetic Algorithms," WSEAS Trans. Comput., vol. 9, no. 9, pp. 1064-1073, 2010

[21] G. Pavai and T. V Geetha, "A Survey on Crossover Operators," ACM Comput. Surv., vol. 49, no. 4, pp. 1-43, 2016.

[22] M. Y. Orong, A. M. Sison, and R. P. Medina, “A new crossover mechanism for genetic algorithm with rank-based selection method," in Proceedings of 2018 5th International Conference on Business and Industrial Research: Smart Technology for Next Generation of Information, Engineering, Business and Social Science, ICBIR, pp. 83-88, 2018.

[23] P. Błażej, M. Wnętrzak, and P. Mackiewicz, "The role of crossover operator in evolutionary-based approach to the problem of genetic code optimization," BioSystems, vol. 150, pp. 61-72, 2016.

[24] A. B. Hassanat, V. B. S. Prasath, M. A. Abbadi, S. A. Abu-Qdari, and H. Faris, "An improved Genetic Algorithm with a new initialization mechanism based on Regression techniques," Inf., vol. 9, no. 7, 2018.

[25] S. Renjith and C. Anjali, "Fitness Function in Genetic Algorithm based Information Filtering - A Survey," in International Conference on Mobility in Computing- ICMiC13, 2013, pp. 80-86.

[26] O. Al Jadaan, L. Rajamani, and C. R. Rao, "Improved selection operator for GA," J. Theor. Appl. Inf. Technol., vol. 4, no. 4, pp. 269-277, 2008.

[27] F. Alabsi and R. Naoum, "Comparison of Selection Methods and Crossover Operations using Steady State Genetic Based Intrusion Detection System," J. Emerg. Trends Comput. Inf. Sci., vol. 3, no. 7, pp. 1053-1058, 2012.

[28] S. L. Yadav and A. Sohal, "Comparative Study of Different Selection Techniques in Genetic Algorithm," Int. J. Sci. Math., vol. 6, no. 3, 2017.

[29] F. Alabsi and R. Naoum, "Comparison of Selection Methods and Crossover Operations using Steady State Genetic Based Intrusion Detection System,” J. Emerg. Trends Comput. Inf. Sci., vol. 3, no. 7, pp. 1053-1058, 2012.

[30] Y. Fan and D. Chen, “Application of Improved Adaptive Genetic Algorithm in Train Energy Saving," in Proceedings of the Fourth International Forum on Decision Sciences, Uncertainty and Operations Research, 2017, pp. 723-736.

[31] H. Rao et al., "Feature selection based on artificial bee colony and gradient boosting decision tree," Appl. Soft Comput. J., vol. 74, pp. 634-642, 2018.

[32] Z. Michalewicz, G. Nazhiyath, and M. Michalewicz, "A note on the usefulness of geometric crossover for numerical optimization problems," Proc. 5th Annu. Conf. Evol. Program., pp. 305-312, 1996.

\section{BIOGRAPHY OF AUTHORS}

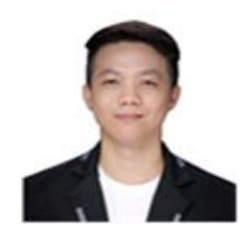

Allemar Jhone P. Delima was born in Surigao City, Philippines. He is a faculty under the College of Engineering and Information Technology of Surigao State College of Technology. He received his B.S. degree from Surigao State College of Technology (SSCT), Surigao City, in 2014 and the master's degree from the same institution in 2016, both in Information Technology. He is currently pursuing his Doctor in Information Technology (DIT) degree in the Technological Institute of the Philippines - Quezon City. His research interest is in Data Mining, Data Analytics, and Machine Learning.

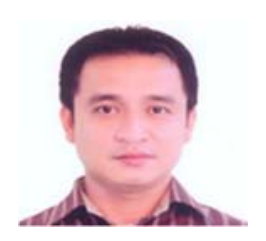

Ariel M. Sison earned his Doctor in Information Technology (DIT) degree at the Technological Institute of the Philippines Quezon City in 2013 and graduated with highest honors. He took up his master's degree in computer science at De La Salle University, Manila in 2006 and obtained his BS in Computer Science at Emilio Aguinaldo College, Manila in 1994. He is currently connected at the School of Computer Studies, Emilio Aguinaldo College in Manila, Philippines. His research interests include data mining and data security.

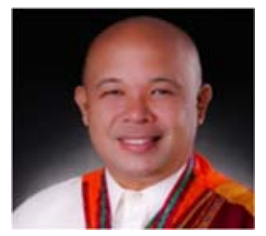

Ruji P. Medina is the dean of the graduate programs of the Technological Institute of the Philippines in Quezon City. He holds a Ph.D. in environmental engineering from the University of the Philippines with a sandwich program at the University of Houston, Texas where he worked on the synthesis of nanocomposite materials. 